Pacific Journal of Mathematics

A REAL INVERSION FORMULA FOR A CLASS OF 


\section{A REAL INVERSION FORMULA FOR A CLASS OF BILATERAL LAPLACE TRANSFORMS}

\section{William R. GAFFeY}

1. Introduction. The Post-Widder inversion formula for unilateral Laplace transformations [1] states that, under certain weak restrictions on $\phi(u)$,

$$
\lim _{k \rightarrow \infty}\left(\frac{k}{c}\right)^{k+1} \frac{1}{k !} \int_{0}^{\infty} \phi(u) u^{k} \exp \left(-k \frac{u}{c}\right) d u=\phi(c)
$$

for any continuity point $c$ of $\phi(u)$.

This formula applies when $\phi(u)$ is defined only for $u \geqq 0$. A similar formula may be deduced if $\phi(u)$ is defined for $u \geqq-a$, for some positive $a$. In such a case, we may let $\phi^{*}(u)=\phi(u-a)$, and we may then use the Post-Widder formula to determine $\phi^{*}(u)$ at the point $u=c+\alpha$. The inversion formula then becomes

$$
\lim _{k \rightarrow \infty}\left(\frac{k}{c+a}\right)^{k+1} \frac{1}{k !} \int_{0}^{\infty} \phi(u-a) u^{k} \exp \left(-k \frac{u}{c+a}\right) d u=\phi(c),
$$

or, if we make the transformation $z=u /(c+a)$,

$$
\lim _{k \rightarrow \infty} \frac{k^{k+1}}{k !} \int_{0}^{\infty} \phi[(c+a) z-a] z^{k} \exp (-k z) d z=\phi(c) .
$$

This suggests that, if $\phi(u)$ is defined for $-\infty<u<\infty$, some sort of limiting form of (1) applies. We shall prove that under suitable restrictions on $\varepsilon$ and on the behavior of $\phi(u)$,

$$
\lim _{k \rightarrow \infty} \frac{k^{k+1}}{k !} \int_{-\infty}^{\infty} \phi\left[\left(c+k^{\varepsilon}\right) z-k^{\varepsilon}\right] z^{k} \exp (-k z) d z=\phi(c) .
$$

2. Remarks. In the following sections $\phi(u)$ will be assumed to be integrable over the interval from $-\infty$ to $\infty$, and $c$ will be assumed to be a continuity point of $\phi(u)$. All limits should be understood to be for increasing values of $k$.

The expression $\delta /\left(c+k^{\varepsilon}\right)$, where $\delta$ and $\varepsilon$ are positive numbers, occurs frequently. It will be denoted by $\delta(k, \varepsilon)$.

Finally, it may be noted that in terms of the Laplace transform of $\phi(u)$ for real $t$,

Received December 7, 1955, and in revised form April 13, 1956. 


$$
f(t)=\int_{-\infty}^{\infty} \phi(u) \exp (-t u) d u,
$$

the inversion formula (2) may be written in the form

$$
\lim \frac{(-1)^{k}}{k !}\left(\frac{k}{c+k^{\varepsilon}}\right)^{k+1} \frac{d^{k}}{d t^{k}}\left[f(t) \exp \left(-t k^{\varepsilon}\right)\right]_{t=k /\left(c+k^{\varepsilon}\right)}=\phi(c) .
$$

3. Preliminary proofs. The results of the following four lemmas will be needed below. Proofs are given for the first two. The second two are proved in a similar way.

Lemma 1. If $n$ is any fixed number and $1 / 3<\varepsilon<1 / 2$, then

$$
\lim k^{n}[1+\delta(k, \varepsilon)]^{k} \exp [-k \delta(k, \varepsilon)]=0 .
$$

Proof. If the logarithm of the expression under the limit sign is expanded in powers of $\delta(k, \varepsilon)$, the sum of two of the terms in the expansion approaches $-\infty$ as $k \rightarrow \infty$, while the sum of the rest of the terms is bounded.

Lemma 2. If $1 / 3<\varepsilon<1 / 2$, then

$$
\lim \frac{k k^{k+1}}{k !} \int_{1}^{1+\delta(k, 3)} z^{k} \exp (-k z) d z=\frac{1}{2} .
$$

Proof. It is well known [1] that

$$
\lim \frac{k{ }^{k+1}}{k !} \int_{1}^{\infty} z^{k} \exp (-k z) d z=\frac{1}{2} .
$$

Therefore, it is sufficient to show that

$$
\lim \frac{k^{k+1}}{k !} \int_{1+\delta(k, \varepsilon)}^{\infty} z^{k} \exp (-k z) d z=0 .
$$

Since $z \exp (-z)$ is a decreasing function of $z$ for $z>1$, the above expression is, for fixed $k$, no larger than

$$
\frac{k^{k+1}}{k !}[1+\delta(k, \varepsilon)]^{k-1} \exp [-(k-1)(1+\delta(k, \varepsilon))] \int_{1+\delta(k, \varepsilon)}^{\infty} z \exp (-z) d z .
$$

By applying Stirling's formula and Lemma 1, we see that the upper bound approaches zero as $k$ increases.

LeMma 3. If $n$ is any fixed number and $0<\varepsilon<1 / 2$, then 


$$
\lim k^{n}[1-\delta(k, \varepsilon)]^{k} \exp [k \delta(k, \varepsilon)]=0
$$

Lemma 4. If $0<\varepsilon<1 / 2$, then

$$
\lim \frac{k^{k+1}}{k !} \int_{1-\delta(k, \varepsilon)}^{1} z^{k} \exp (-k z) d z=\frac{1}{2} .
$$

\section{The inversion formula.}

THEOREM. If

$$
\left|\int_{-\infty}^{-\infty} \phi(z) d z\right| \leqq A \exp \left(-d x^{2+\alpha}\right)
$$

for some positive quantities $A, d$, and $\alpha$, and if

$$
\max (1 / 3,1 /(2+\alpha))<\varepsilon<1 / 2,
$$

then

$$
\lim I_{k}=\lim \frac{k^{k+1}}{k !} \int_{-\infty}^{\infty} \phi\left[\left(c+k^{\varepsilon}\right) z-k^{\varepsilon}\right] z^{k} \exp (-k z) d z=\phi(c)
$$

Proof. For any $\delta>0$, the infinite interval may be partitioned into the four subintervals $(-\infty, 1-\delta(k, \varepsilon)),(1-\delta(k, \varepsilon), 1),(1,1+\delta(k, z))$, and $(1+(k, \varepsilon), \infty) . \quad I_{k}$ may be considered as the sum of four integrals over these intervals, so that we may write

$$
I_{k}=I_{k}^{(1)}+I_{k}^{(2)}+I_{k}^{(3)}+I_{k}^{(4)} .
$$

$I_{k}^{(1)}$ is understood to represent the integral over $(-\infty, 1-\delta(k, \varepsilon))$ etc.

$$
\left|I_{k}-\phi(c)\right| \leqq\left|I_{k}^{(1)}\right|+\left|I_{k}^{(2)}-\frac{\phi(c)}{2}\right|+\left|I_{k}^{(3)}-\frac{\phi(c)}{2}\right|+\left|I_{k}^{(4)}\right|
$$

We prove first that $I_{k}^{(1)}$ and $I_{k}^{(4)}$ approach zero as $k \rightarrow \infty$. For $I_{k}^{(1)}$, consider first the integral over the interval from 0 to $1-\delta(k, \varepsilon)$. The function $z \exp (-z)$ attains its maximum at the upper endpoint. Therefore an upper bound for the absolute value of this portion of the expression is

$$
\frac{k^{k+1}}{k !}[1-\delta(k, \varepsilon)]^{k} \exp [-k+k \delta(k, \varepsilon)] \int_{0}^{1-\delta(k, \varepsilon)}\left|\phi\left[\left(c+k^{\varepsilon}\right) z-k^{\varepsilon}\right]\right| d z,
$$

which approaches zero by Stirling's formula and Lemma 3.

Consider now the integral over the interval from $-\infty$ to 0 . Integrating by parts, we find that it is equal to 


$$
-\frac{1}{c+k^{\varepsilon}} \frac{k^{k+2}}{k !} \int_{-\infty}^{0} F\left[\left(c+k^{\varepsilon}\right) z-k^{\varepsilon}\right] z^{k+1}(1-z) \exp (-k z) d z
$$

where $F(z)=\int_{-\infty}^{z} \phi(u) d u$. Note that, by the assumption on $F(z)$,

$$
\left|F\left[\left(c+k^{\varepsilon}\right) z-k^{\varepsilon}\right]\right| \leqq A \exp \left[-d\left\{-\left(c+k^{\varepsilon}\right) z+k^{\varepsilon}\right\}^{2+\alpha}\right],
$$

which is in turn equal to or less than

$$
A \exp \left[d z\left(c+k^{\varepsilon}\right) k^{\varepsilon(1+\alpha)}\right] .
$$

The result of the integration by parts may be written as the difference between two integrals, the first containing $z^{k-1}$ and the second containing $z^{k}$. The first integral is no greater in absolute value than

$$
\frac{A}{\left(c+k^{\varepsilon}\right)} \frac{k^{k+2}}{k !} \int_{-\infty}^{0}\left|z^{k-1}\right| \exp \left[z\left\{d\left(c+k^{\varepsilon}\right) k^{\varepsilon(1+\alpha)}-k\right\}\right] d z \text {. }
$$

Since $\varepsilon(2+\alpha)>1$, the coefficient of $z$ in the exponent above is positive for sufficiently large $k$. Therefore, after some manipulation, this upper bound can be shown to be equal to

$$
\frac{A}{\left(c+k^{z}\right)} k^{k+2} \cdot \frac{\Gamma(k)}{\left[d\left(c+k^{\varepsilon}\right) k^{\varepsilon(1+\alpha)}-k\right]^{k}},
$$

which approaches zero as $k \rightarrow \infty$.

By the same argument, the second integral approaches zero, so that $\lim I_{k}^{(1)}=0$.

For $I_{k}^{(4)}$, observe that since $z \exp (-z)$ is a decreasing function of $z$ for $z>1$, the expression has the following upper bound for its absolute value :

$$
\frac{k^{k+1}}{k !}[1+\delta(k, \varepsilon)]^{k} \exp [-k-k \delta(k, \varepsilon)] \int_{1+\delta(k, \varepsilon)}^{\infty}\left|\phi\left[\left(c+k^{\varepsilon}\right) z-k^{\varepsilon}\right]\right| d z .
$$

Since the integral is bounded, the whole upper bound approaches zero by virtue of Stirling's formula and Lemma 1.

We now prove that

$$
\left|\lim I_{k}^{(3)}-\frac{1}{2} \phi(c)\right|<\frac{1}{2} \eta
$$

for any $\eta>0$. By Lemma 2, it is sufficient to show that

$$
\left|\lim \frac{k^{k+1}}{k !} \int_{1}^{1+\delta(k, \varepsilon)}\left\{\phi\left[\left(c+k^{\varepsilon}\right) z-k^{\varepsilon}\right]-\phi(c)\right\} z^{k} \exp (-k z) d z\right|<\frac{\eta}{2} .
$$


Since $c$ is a continuity point of $\phi(u)$, there is a $\delta>0$ such that if $\left|\left(c+k^{\varepsilon}\right) z-k^{\varepsilon}-c\right|<\delta$, that is, if $|z-1|<\delta(k, \varepsilon)$, then

$$
\left|\phi\left[\left(c+k^{\varepsilon}\right) z-k^{\varepsilon}\right]-\phi(c)\right|<\eta \text {. }
$$

For such a $\delta$, the absolute value of the expression above is equal to or less than

$$
\eta \lim \frac{k^{k+1}}{k !} \int_{1}^{1+\delta(k, \varepsilon)} z^{k} \exp (-k z) d z=\frac{\eta}{2}
$$

By the use of Lemma 4, it may be shown in a similar way that

$$
\left|\lim I_{k}^{(2)}-\frac{1}{2} \phi(c)\right|<\frac{1}{2} \eta \text {. }
$$

Putting together these results, we have $\left|\lim I_{k}-\phi(c)\right|<\eta$ for any $\eta>0$, which proves the theorem.

\section{REFERENCE}

1. C. V. Widder, Inversion of the Laplace transform and the related moment problem, Trans. Amer. Math. Soc. 36 (1934), 107-200.

UNIVERSITY OF CALIFORNIA, BERKELEY 



\section{PACIFIC JOURNAL OF MATHEMATICS}

EDITORS

H. L. ROYDEN

Stanford University

Stanford, California

R. A. Beaumont

University of Washington

Seattle 5 , Washington
A. R. Whiteman

University of Southern California

Los Angeles 7, California

E. G. Straus

University of California

Los Angeles 24, California

\section{ASSOCIATE EDITORS}
E. F. BECKENBACH
C. E. BURGESS
M. HALL
E. HEWITT
A. HORN
V. GANAPATHY IYER
R. D. JAMES
M. S. KNEBELMAN

L. NACHBIN

I. NIVEN

G. SZEKERES

T. G. OSTROM

M. M. SCHIFFER
F. WOLF

K. YOSIDA

\section{SUPPORTING INSTITUTIONS}

UNIVERSITY OF BRITISH COLUMBIA

CALIFORNIA INSTITUTE OF TECHNOLOGY

UNIVERSITY OF CALIFORNIA

MONTANA STATE UNIVERSITY

UNIVERSITY OF NEVADA

OREGON STATE COLLEGE

UNIVERSITY OF OREGON

UNIVERSITY OF SOUTHERN CALIFORNIA

\author{
STANFORD UNIVERSITY \\ UNIVERSITY OF UTAH \\ WASHINGTON STATE COLLEGE \\ UNIVERSITY OF WASHINGTON \\ AMERICAN MATHEMATICAL SOCIETY \\ CALIFORNIA RESEARCH CORPORATION \\ HUGHES AIRCRAFT COMPANY \\ THE RAMO-WOOLDRIDGE CORPORATION
}




\section{Pacific Journal of Mathematics}

\section{Vol. 7, No. $1 \quad$ January, 1957}

Richard Davis Anderson, Zero-dimensional compact groups of

homeomorphisms ................................... 797

Hans-Joachim Bremermann, Holomorphic functionals and complex

convexity in Banach spaces........................... 811

Hugh D. Brunk, G. M. Ewing and W. R. Utz, Minimizing integrals in

certain classes of monotone functions ................. 833

Philip David, Uniqueness theory for asymptotic expansions in general

regions ...................................... 849

Paul Erdős and Harold Nathaniel Shapiro, On the least primitive root of a

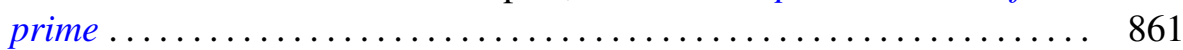

Watson Bryan Fulks, Regular regions for the heat equation ........... 867

William Robert Gaffey, A real inversion formula for a class of bilateral

Laplace transforms ................................ 879

Ronald Kay Getoor, On characteristic functions of Banach space valued random variables ................................. 885

Louis Guttman, Some inequalities between latent roots and minimax (maximin) elements of real matrices ...................... 897

Frank Harary, The number of dissimilar supergraphs of a linear graph .... 903

Edwin Hewitt and Herbert S. Zuckerman, Structure theory for a class of convolution algebras .................................. 913

Amnon Jakimovski, Some Tauberian theorems . . . . . . . . . . . . . . . . . 943

C. T. Rajagopal, Simplified proofs of "Some Tauberian theorems" of Jakimovski................................

Paul Joseph Kelly, A congruence theorem for trees ................. 961

Robert Forbes McNaughton, Jr., On the measure of normal formulas...... 969

Richard Scott Pierce, Distributivity in Boolean algebras .............. 983

Calvin R. Putnam, Continuous spectra and unitary equivalence ......... 993

Marvin Rosenblum, Perturbation of the continuous spectrum and unitary

equivalence................................... 997

V. N. Singh, Certain generalized hypergeometric identities of the

Rogers-Ramanujan type.......................

Peter Swerling, Families of transformations in the function spaces $H^{p} \ldots \ldots 1015$ 